\title{
Is current disruption associated with an inverse cascade?
}

\author{
Z. Vörös ${ }^{1}$, A. Runov ${ }^{2}$, M. P. Leubner ${ }^{1}$, W. Baumjohann ${ }^{3}$, and M. Volwerk ${ }^{3}$ \\ ${ }^{1}$ Institute of Astro- and Particle Physics, University of Innsbruck, Innsbruck, Austria \\ ${ }^{2}$ UCLA, Los Angeles, USA \\ ${ }^{3}$ Space Research Institute, Austrian Academy of Sciences, Graz, Austria
}

Received: 18 February 2010 - Revised: 24 May 2010 - Accepted: 9 June 2010 - Published: 16 June 2010

\begin{abstract}
Current disruption (CD) and the related kinetic instabilities in the near-Earth magnetosphere represent physical mechanisms which can trigger multi-scale substorm activity including global reorganizations of the magnetosphere. Lui et al. (2008) proposed a CD scenario in which the kinetic scale linear modes grow and reach the typical dipolarization scales through an inverse cascade. The experimental verification of the inverse nonlinear cascade is based on wavelet analysis. In this paper the Hilbert-Huang transform is used which is suitable for nonlinear systems and allows to reconstruct the time-frequency representation of empirical decomposed modes in an adaptive manner. It was found that, in the Lui et al. (2008) event, the modes evolve globally from high-frequencies to low-frequencies. However, there are also local frequency evolution trends oriented towards high-frequencies, indicating that the underlying processes involve multi-scale physics and non-stationary fluctuations for which the simple inverse cascade scenario is not correct.
\end{abstract}

\section{Introduction}

A key element of substorm physics is an enhanced transport of magnetic flux from the dayside magnetopause over the poles to the magnetotail during the growth phase of substorms (Baumjohann et al., 1999). In the tail the magnetic energy is accumulated and subsequently abruptly released during the expansion phase. In general, substorms are associated with multi-scale processes including electron(tens of kms), ion- (hundreds of kms), MHD- ( $\geq$ thousands of kms) scales and global reconfigurations of the magnetosphere (Nakamura et al., 2006; Laitinen et al., 2007). For this reason, the identification of location(s) and timing of

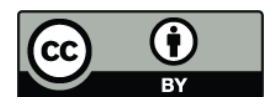

Correspondence to: Z. Vörös

(zoltan.voeroes@uibk.ac.at) substorm triggering mechanisms was not fully conclusive. Magnetic reconnection (MR), converting magnetic energy into kinetic energy of accelerated plasma and energetic particles at 20 to $30 R_{\mathrm{E}}$, could explain many substorm signatures, such as magnetic field dipolarization due to flow breaking at the barrier of strong dipolar field (at $\sim 10 R_{\mathrm{E}}$ ), flux pile-up and the tailward motion of dipolarization front, the formation of substorm current wedge, and the associated auroral and ground based activities (Baumjohann et al., 1999). On the other hand, the substorm triggering scenario called current disruption (CD) invokes kinetic instability processes leading to cross-tail CD (current reduction), dipolarization, formation of the substrom current wedge in the near -Earth region at $\sim 10 R_{\mathrm{E}}$ and other auroral and ground-based substorm signatures (Lui, 2004). The CD scenario offers a possibility to explain both the dipolarization and the tailward movement of dipolarization front without invoking reconnection associated flow breakings or flux pile-up. In fact, some dipolarizations were observed without local Earthward plasma flows (Lui et al., 2008). Though the multi-spacecraft timing observations from THEMIS spacecraft indicate predominant substorm triggering due to MR (Angelopoulos, 2008), both mid-tail (MR) and near-Earth (CD) processes can contribute to the global substorm activity. For example, MR associated Earthward flows might trigger near-Earth CD (Runov et al., 2008).

$\mathrm{CD}$ is associated with kinetic instabilities in rather localized regions. It was shown by Lui (2004) that a local kinetic theory of instabilities can account for the excited waves exhibiting large growth for long enough time even within a thin current sheet. The stability analysis of cross-field ion-drift driven instabilities based on a two-fluid approach also showed that waves initially excited near the ion cyclotron frequency $(\sim 0.1 \mathrm{~Hz}, 10 \mathrm{~s})$ can grow and reach the typical time scales associated with dipolarization $(\sim 0.006$ $0.008 \mathrm{~Hz}, 120-170 \mathrm{~s}$ ). It was suggested (Lui et al., 2008) that the corresponding experimental signature would be a

Published by Copernicus Publications on behalf of the European Geosciences Union and the American Geophysical Union. 




Fig. 1. (a) Test signal: sine wave + sudden jump; (b) Wavelet analysis of the test signal; (c) Hilbert-Huang analysis of the test signal.

developing inverse cascade in wavelet time-frequency representation, i.e., the evolution of wave modes from higher (shorter) to lower (longer) frequencies (time scales) in time. Conversely, forward cascades in turbulence transfer energy from large scales to small scales. The dynamics, driving sources and dissipation of turbulent fluctuations were reviewed by Borovsky and Funsten (2003). In the Earth's plasma sheet turbulence was also observed within reconnection associated bursty flows (Vörös et al., 2004). However, developing turbulence was not identified, possibly because of the short duration of events or due to the observation of already fully developed turbulence with broad-band fluctuations (with no time evolution of characteristic frequencies). Observations of a developing turbulence would help us to identify the spatial or temporal scales of turbulent drivers (the scales of energy input) and to recognize the direction of turbulent cascades (forward or inverse). Anyhow, this would require to observe the associated fluctuations over the characteristic scales of turbulent drivers first, followed by the development of multi-scale fluctuations over the inertial range of scales.

\section{Limitations of the wavelet analysis}

While the experimental identification of inverse cascades would be crucial for understanding the real growth rate of excited kinetic instability modes, the detection based on wavelet analysis has to be interpreted rather carefully. The main reason is that the widely used basic wavelet analysis is non-adaptive (Huang et al., 1998). Once the basic wavelet is chosen it is used for the whole data set. This is not a problem when observations with gradual frequency changes, chirp signals, or wave trains are analyzed. Actually, the harmonic signals used to test the wavelet identifica- tion of inverse cascade features by Lui et al. (2008) belong to this category of well treatable signals. However, sudden jumps (non-stationarity) or nonlinearity can generate spurious time-frequency-energy distributions in a wavelet representation. The basic wavelet transform is also linear (Huang et al., 1998). Nevertheless, wavelets can be used successfully for the multi-scale analysis of nonlinear systems (KijewskiCorrea and Kareem, 2006). The results of wavelet analysis, however, heavily depend on the mother wavelet, proper resolution, scale discretization (Kijewski-Correa and Kareem, 2006), resulting in non-adaptiveness for multi-component non-stationary data. The effect of non-stationarity is demonstrated in Fig. 1a, b. The test data consisting of a single sine wave with period $1 \mathrm{~s}$ and a sudden jump (Fig. 1a) is analyzed using the Morlet wavelet. The time-wavelet period-energy distribution (wavelet scalogram) in Fig. 1b is showing that the time localization of the sine wave (between 4 and $5 \mathrm{~s}$ ) is good only at wavelet periods near $\sim 0.125 \mathrm{~s}$. The time localization is rather poor at the wavelet period of $1 \mathrm{~s}$, which is the actual sine wave period. Using a different, optimized wavelet might lead to a slightly better localization of the sine wave. Certainly, a mother wavelet cannot be equally good for an unknown combination of periodic or stochastic components in a time series.

Sudden jumps lead to broad-band energy distributions (Fig. 1b). Since for higher frequencies the basic wavelet is more localized, the sudden jump at Time $=9 \mathrm{~s}$ is well localized in time over small wavelet period ranges. The time localization worsens towards longer periods where the basic wavelet is more stretched. The non-stationarity also induces spurious periodicities in the time-period plane, in our case between Time $=7-8 \mathrm{~s}$, where the test signal contains no fluctuations at all. Actually, it can be even worse than that. The wavelet identification of scales (periods) can be entirely misleading. For example, Huang et al. (1998) argue that a change occurring over large scales locally in time will appear in the corresponding time-period distribution over small scales, due to the basic wavelet resolution. That is, a localized large-scale change (non-stationarity) manifests itself over small scales in the wavelet spectrum.

\section{The Hilbert-Huang method}

To be able to identify inverse cascade features we need a method which is adaptive and suitable for non-stationary and nonlinear analysis of multi-scale data. The Hilbert transform (HT) represents a convolution of time series $X(t)$ with time $1 / t$, therefore accentuates the local properties of $X(t)$. HT is defined as (Huang et al., 1998)

$Y(t)=\frac{1}{\pi} P \int_{-\infty}^{\infty} \frac{X(\tau)}{t-\tau} d \tau$ 


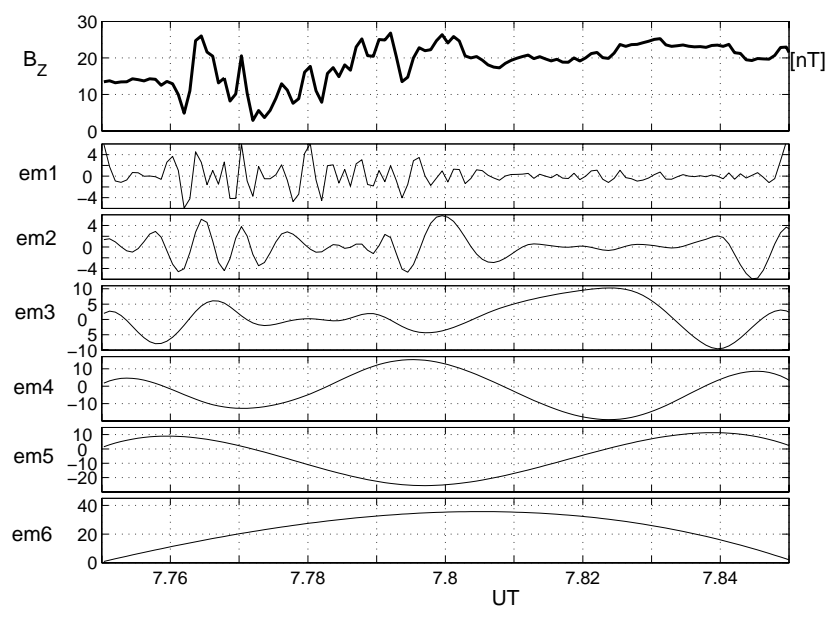

Fig. 2. Top subplot: magnetic field $B_{Z}$ component; Bottom subplots: empirical modes; emis, for $i=1,2, \ldots 6$.

where $P$ is the Cauchy principal value of the integral. $X(t)$ and $Y(t)$ are orthogonal and form a complex conjugate pair

$Z(t)=X(t)+i Y(t)=a(t) e^{i \Theta(t)}$

$a(t)$ and $\Theta(t)$ are the instantenous amplitude and phase, respectively. The instantenous angular frequency $\omega(t)$ is the time derivative of $\Theta(t)$. HT is known for a long time, but the practical value of instantenous frequency $\omega(t)$ was questioned, because of non-unique local values. Local uniqueness can be achieved by putting some constraints on the data (Cohen, 1995). Huang et al. (1998) proposed a practical decomposition algorithm, called empirical mode decomposition (EMD), which removes the ambiguity in determination of $\omega$. The extracted empirical modes $(\mathrm{ems})$ fulfill certain conditions without any loss of essential nonlinear features of the original data and also ensure adaptiveness to local signal characteristics (Huang et al., 1998). The EMD procedure fits splines to local maxima and minima of $X(t)$, finds the mean $X_{\mathrm{m}}$ from the two spline functions and computes the residuals $X_{\mathrm{r}}(t)=X(t)-X_{\mathrm{m}}(t)$. These steps are iterated until a threshold is reached. The first mode is given by $\operatorname{em} 1(t)=X_{\mathrm{m}}(t)$. The next mode is found in the same way as em 1 , but replacing the original data $X(t)$ with $X(t)-e m 1(t)$. Successive emi modes are found by repeating the whole process, until the last residue contains only a simple trend. Even complex signals can be decomposed to a finite number of emis. For example, HT based on the EMD was successfully applied to intermittent turbulence (Huang et al., 2008) just recently. The extracted em modes represents narrow band stationary Gaussian processes. Moreover, the modes are locally symmetric relative to the envelopes defined by the local maxima and minima. This means that the local means are not computed over a time window or a local time scale, but are obtained directly from the local values of the envelopes.

The instantenous frequency is determined by taking HT of each $\operatorname{emi}(t)$. The time and frequency localization is demon-

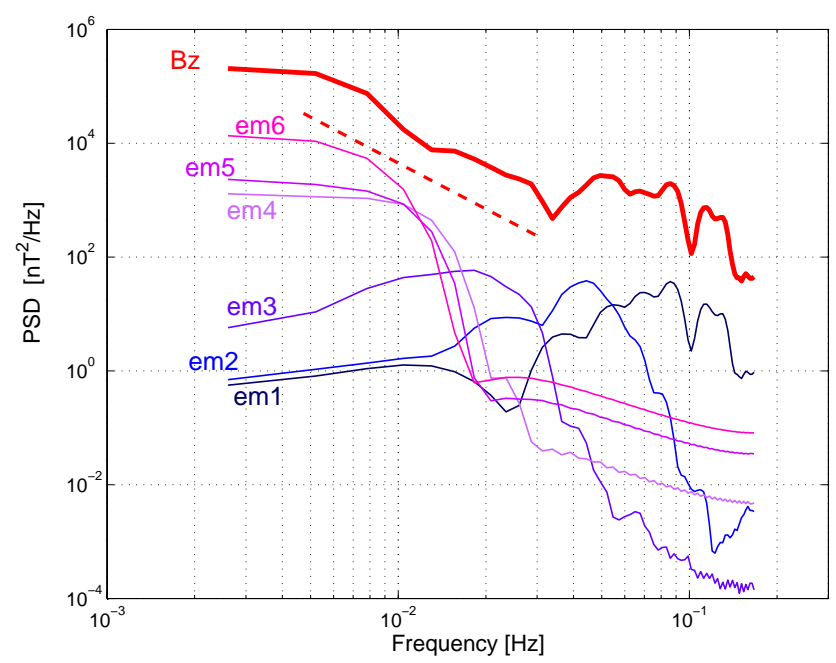

Fig. 3. Power spectral density for magnetic field (red thick line) and empirical modes; the dashed red line shows the scaling region over the inverse cascade frequency range.

strated for the test signal in Fig. 1c. The test signal was decomposed into three emis (not shown). Contrary to wavelet results (Fig. 1b) the localization is rather good, without spurious energy distributions in the time-frequency distribution. Now, we are going to apply the Hilbert-Huang method to the event studied by Lui et al. (2008), proclaimed to be an example of inverse cascade observed by the wavelet method.

\section{Identification of an inverse cascade}

$\mathrm{CD}$ and the associated dipolarization was observed by the THEMIS A spacecraft at $X_{\mathrm{GSM}} \sim-8 R_{\mathrm{E}}$, between 07:45 and 07:50 UT on 29 January 2008. (In what follows, we will use decimal hours). Since the event occurred near a substorm expansion onset, $\mathrm{CD}$ is considered to be a substorm triggering agent. The inverse cascade was found to be most remarkable in the magnetic component $B_{\mathrm{Z}}$ (Lui et al., 2008).

The top panel in Fig. 2 shows the $B_{\mathrm{Z}}$ component of the magnetic field. Using the Hilbert-Huang method described above six emis were found which represent the decomposition of $B_{\mathrm{Z}}$ into "monocomponent" modes. The modes are shown in the subsequent subplots in Fig. 2. In Fig. 3 the power spectral density (PSD) is depicted, computed for $B_{\mathrm{Z}}$ (thick red line, for a better visibility shifted) and em $1-6$. The monocomponent modes are narrow-band fluctuations, having finite bandwidth in PSD. For example, em 2 exhibits the maximum power around the frequency $\sim 4.5 \times 10^{-2} \mathrm{~Hz}$. Nevertheless, Fig. 3 represents only a rough demonstration of monocomponent modes. The modes in PSD correspond to a global description based on the Fourier transform. The monocomponent modes identified through the HilbertHuang method differ due to the localized constraints introduced in their determination explained above in Sect. 3. In 

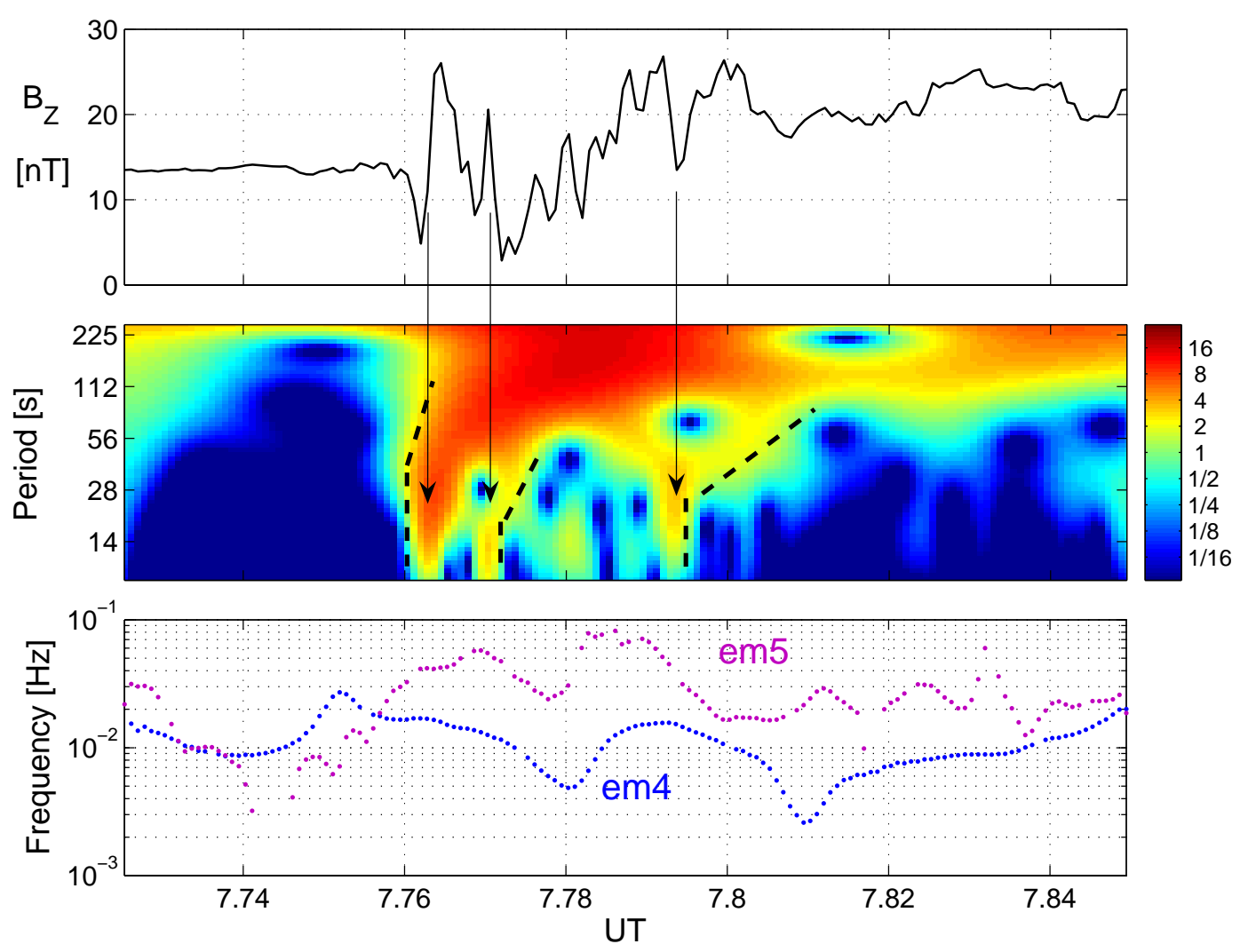

Fig. 4. Top: magnetic field time series; Middle: Wavelet time-period-energy representation; Bottom: Hilbert-Huang time-instantenous frequency representation; Thick dashed lines in the middle indicate the wavelet time-scale evolution in time; Line arrows show the locations of short time-scale (high-frequency) activations occurring simultaneously with the largest jumps in $B_{\mathrm{Z}}$.

spite of the finite bandwith the Hilbert-Huang modes can exhibit non-stationarity through frequency and amplitude modulation (Huang et al., 1998).

Following the Lui et al. (2008) findings the inverse cascade should operate roughly over the frequency range from $10^{-1}$ to $5 \cdot 10^{-3} \mathrm{~Hz}$. The magnetic field PSD shows scaling (thick red dashed line) over a narrower range from $3.10^{-2}$ to $5 \cdot 10^{-3} \mathrm{~Hz}$. Though we do not intend to identify the inverse cascade on the basis of PSD, this scaling region could be associated with the developing nonlinear interactions. The power of $e m 1-3$ modes over the scaling region is by $2-4$ orders of magnitude smaller than the power of em 4-6 modes. Moreover, the em 6 mode contains only a smooth trend, therefore, we will investigate em $4-5$ further.

Figure 4 compares the wavelet time-period-energy spectrum (middle subplot) and the Hilbert-Huang time-frequency spectrum (bottom subplot). $B_{Z}$ is in the top subplot. There are three vertical and inclined dashed lines in the wavelet plot. The lines are guides for eyes to see the frequency trend of activity in time. At least in three cases, there is an increased wavelet power which appears first at shorter time scales near the ion gyroperiod $(\sim 10 \mathrm{~s})$. Power over longer time scales appears later. Our wavelet representation of $B_{\mathrm{Z}}$ is slightly different than the one published by Lui et al. (2008). Nevertheless, the wavelet representation shows the same qualitative features as the wavelet analysis in Lui et al. (2008) paper: increasing wavelet power bridging gradually the scales between the ion gyroperiod $(\sim 10 \mathrm{~s})$ and typical dipolarization time $(>100 \mathrm{~s})$. It is easy to notice that the enhanced small-scale wavelet power (indicated by dashed vertical lines in Fig. 4) and the largest amplitude jumps in $B_{\mathrm{Z}}$ occur simultaneously (vertical arrow lines). The dubious small-scale activity can occur due to a local sharp change (like in Fig. 1), which might have nothing to do with the inverse cascade. Having in mind the limitations of the wavelet method, the uncertainty connected with sharp jumps in the data cannot be removed. Therefore, a wavelet independent test is needed. The bottom subplot in Fig. 4, corresponding to the Hilbert-Huang time-frequency spectrum of em 4 and em 5 empirical modes (these are within the PSD scaling region, see Fig. 3), seems to substantiate the idea of multiscale physics. We note, the emi spectra cannot be interpreted roughly before 07.74 and after 07.84 UT because of interval finite-size effects. The first high-frequency activation (at $\sim 3.10^{-2} \mathrm{~Hz}$ after $07.75 \mathrm{UT}$ ) in em 4 occurs simultaneously with the beginning of small amplitude fluctuations in $B \mathrm{Z}$. 
There is no increased power visible in the wavelet spectrum at around $07.75 \mathrm{UT}$ in Fig. 4, neither in the wavelet spectrum of Lui et al. (2008), which could be associated with those $B_{\mathrm{Z}}$ fluctuations. In em 4 the frequency decreases to $5.10^{-3} \mathrm{~Hz}$ at $07.78 \mathrm{UT}$ then, after a short return to $\sim 10^{-2} \mathrm{~Hz}$, it falls down to $\sim 3.10^{-3} \mathrm{~Hz}$ at 07.81 UT. Overall, the instantenous em 4 time scales change from 30 to $330 \mathrm{~s}$ as time proceeds. During the interval of interest, em 5 varies between $8.10^{-2}$ and $10^{-2}(10-100 \mathrm{~s})$. There are a few correlations between the wavelet and Hilbert-Huang spectra in Fig. 4. For example, the enhanced small-scale activity in wavelet spectra is associated with high-frequency fluctuations in $\mathrm{em} 5$ between 07.76 and 07.8 UT. The first two local peaks in $e m 5$ seem to correlate with the first two small-scale activations in the wavelet spectra between 07.76 and 07.77 UT. On the other hand, the local peak in em5 between 07.78 and 07.79 UT has no counterpart in the wavelet spectra. Moreover, there are low-frequency valleys in between high-frequency local peaks, which are related to the occurrence of multi-scale interactions more complicated than a simple inverse cascade. In contrary to the wavelet method the Hilbert-Huang results indicate that the inverse cascade picture cannot fully explain the observed multi-scale fluctuations. This does not necessarily mean that the linkage between local kinetic instabilities and large-scale processes is negligible.

\section{Conclusions}

In this paper we have shown that the typical limitations of wavelet methodology make the identification of $\mathrm{CD}$ associated inverse cascades doubtful and misleading. The observations of $\mathrm{CD}$ associated fluctuations are short in time, the magnetic field changes are highly dynamic. As it was outlined in the paper, the Hilbert-Huang method is more suitable for nonstationary and nonlinear processes. Its adaptiveness allows to compute straightforwardly the instantenous frequency and reproduce the time-frequency representation of the time series based on decomposed empirical modes. In qualitative agreement with the wavelet results, the time evolution of instantenous frequency shows a general trend of raising magnetic fluctuations from high-frequencies to low-frequencies. However, the details of multi-scale fluctuations and the time locations of high-frequency activations are rather different in the wavelet and the Hilbert-Huang spectra. In the latter, there are also intervals where the frequency locally increases with time. In this paper we put a particular emphasis on the differences between the wavelet and Hilbert-Huang representations, demonstrating that for the $\mathrm{CD}$ associated non-stationary magnetic fluctuations the wavelet approach can lead to misleading conclusions. We believe the more adaptive Hilbert-Huang approach can reconstruct the time evolution of instantenous frequency better, therefore, the identification of turbulent cascades occurring over the appropriate frequency ranges could be more straightforward. Since we were not able to identify a simple inverse cascade from the data, the contribution of other physical processes to the observed fluctuations cannot be ruled out. For example, large-scale variations in the curvature of the ambient magnetic field lines driven by ballooning instability prior to substorm associated dipolarization onsets can lead to fluctuations over the frequency range $10^{-1}-10^{-2} \mathrm{~Hz}$ (Saito et al., 2008). This is also the frequency range over which the inverse cascade proposed by Lui et al. (2008) should occur. Global instabilities, including magnetosphere-ionosphere coupling (Kan, 2007) can also drive low-frequency waves. As a matter of fact, the identification of low-frequency fluctuations is rather difficult or impossible when the whole length of event observations is comparable to the period of waves. It is even more difficult to detect the non-stationarity of low-frequency components over short intervals using wavelets. In this paper it is shown that the low-frequency components of fluctuations (em $4-5$ modes in Figs. 3 and 4) are non-stationary during the current disruption event of Lui et al. (2008). As it was mentioned in Sect. 2 non-stationarity of large-scale lowfrequency waves manifests itself over high-frequencies in the wavelet spectrum, therefore the wavelet observations cannot be fully trusted.

There exist also physical reasons which makes the identification of turbulent cascades difficult. Inverse or forward cascades in turbulence are related to local mode interactions in the Fourier space. It is known that in the presence of a uniform magnetic field magnetohydrodynamic turbulence is neither isotropic nor local in Fourier space (Alexakis, 2007). There exist experimental indications that such nonlocal interactions can occur near plasma boundaries with developed gradients (Vörös et al., 2007). The near-Earth location where $\mathrm{CD}$ and magnetic field dipolarization occurs represents a dynamically changing boundary between dipolar and more stretched field lines. Spatial and temporal changes can be mixed up in one-spacecraft in-situ observations and the multi-scale signatures indicating the occurrence of an inverse cascade in the frequency domain misleading.

Acknowledgements. We acknowledge NASA contract NAS502099 and V. Angelopoulos for use of data from the THEMIS Mission. We thank K. H. Glassmeier and U. Auster for the use of FGM data provided under the lead of the Technical University of Braunschweig and with financial support through the German Ministry for Economy and Technology and the German Center for Aviation and Space (DLR) under contract 50 OC 0302. The work of Z.V. and M.P.L was supported by the Austrian "Fonds zur Förderung der wissenschaftlichen Forschung" under project P20131-N16.

Edited by: J. Büchner

Reviewed by: W. M. Macek and another anonymous referee 


\section{References}

Alexakis A.: Nonlocal phenomenology for anisotropic magnetohydrodynamic turbulence, Astrophys. J., 667, L93-L96, 2007.

Angelopoulos, V., McFadden, J. P., Larson, D., Carlson, Ch. W., Mende, S. P., Frey, H., Phan, T., Sibeck, D. G., Glassmeier, K.H., Auster, U., Donovan, E., Mann, I. R., Rae, I. J., Russel, Ch. T., Runov, A., Zhou, X.-Z., and Kepko, L.: Tail reconnection triggering substorm onset, Science, 321, 931-935, 2008.

Baumjohann, W., Hesse, M., Kokubun, S., Mukai, T., Nagai, T., and Petrukovich, A. A.: Substorm, dipolarization and recovery, J. Geophys. Res., 104, 24995-25000, 1999.

Borovsky, J. E. and Funsten, H. O.: MHD turbulence in the Earth's plasma sheet: dynamics, dissipation and driving, J. Geophys. Res., 108, 1284, doi:10.1029/2002JA009625, 2003.

Cohen, L.: Time-frequency analysis, Prentice Hall., New York, 1995.

D’Amicis, R., Bruno, R., and Bavassano, B.: Is geomagnetic activity driven by solar wind turbulence?, Geophys. Res. Lett., 34, L05108, doi:10.1029/2006GL028896, 2007.

Gonzales, W. D. and Tsurutani, B. T.: Criteria for interplanetary parameters causing intense magnetic storms $\left(D_{s t}<-100 \mathrm{nT}\right)$, Planet. Space Sci., 35, 1101-1109, 1987.

Huang, N. E., Shen, Z., Long, S. R., Wu, M. C., Shih, H. H., Zheng, Q., Yen, N.-C., Tung, C. C., and Liu, H. H.: The empirical mode decomposition and the Hilbert spectrum for nonlinear and nonstationary time series, Proc. R. Soc. London, Ser. A, 454, 903995, 1998.

Huang, Y. X., Schmitt, F. G., Lu, Z. M., and Liu, Y. L.: An amplitude-frequency study of turbulent scaling intermittency using empirical mode decomposition and Hilbert spectral analysis, Europhys. Lett., 84, 40010, doi:10.1209/0295-5075/84/40010, 2008.

Kan, J. R.: On the formation of near-Earth X-line at substorm expansion onset, J. Geophys. Res., 112, A01207, doi:10.1029/2006JA012011, 2007.

Kiejewski-Correa, T. and Kareem, A.: Efficacy of Hilbert and wavalet transforms for time-frequency analysis, J. Engineer. Mech., 132, 1037, doi:10.1061/(ASCE)07339399(2006)132:10(1037), 2006.
Laitinen, T. V., Nakamura, R., Runov, A., Rème, H., and Lucek, E. A.: Global and local disturbances in the magnetotail during reconnection, Ann. Geophys., 25, 1025-1035, 2007, http://www.ann-geophys.net/25/1025/2007/.

Lui, A. T. Y.: Potential plasma instabilities for substorm expansion onset, Space Sci. Rev., 113, 127-206, 2004.

Lui, A. T. Y., Volwerk, M., Dunlop, M. W., Alexeev, I. V., Fazakerley, A. N., Walsh, A. P., Lester, M., Grocott, A., Moukis, C., Henderson, M. G., Kistler, L. M., Shen, C., Shi, J. K., Zhang, T. L., and Rème, H.: Near-Earth substorm features from multiple satellite observations, J. Geophys. Res., A07S26, doi:10.1029/2007JA012738, 2004.

Lui, A. T. Y., Yoon, P. H., Mok, C., and Ryu, C.-M.: Inverse cascade feature of current disruption, J. Geophys. Res., 113, A00C06, doi:10.1029/2008JA013521, 2008.

Nakamura, R., Baumjohann, W., Asano, Y., Runov, A., Balogh, A., Owen, C. J., Fazakerley, A. N., Fujimoto, M., Klecker, B., and Rème, H.: Dynamics of thin current sheets associated with magnetotail reconnection, J. Geophys. Res., 111, A11206, doi:10.1029/2006JA011706, 2006.

Runov, A., Angelopoulos, V., Zhou, X.-Z., Voronkov, I. O., Kubyshkina, M. V., Nakamura, R., Carlson, C. W., Frey, H. U., McFadden, J. P., Larson, D., Mende, S. B., Glassmeier, K.-H., Auster, U., and Singer, H. J.: Multipoint in situ and groundbased observations during auroral intensifications, J. Geophys. Res., 113, A00C07, doi:10.1029/2008JA013493, 2008.

Saito, M. H., Miyashita, Y., Fujimoto, M., Shinohara, I., Saito, Y., Liou, K., and Mukai, T.: Ballooning mode waves prior to substorm-associated dipolarizations: Geotail observations, Geophys. Res. Lett., 35, L07103, doi:10.1029/2008GL033269, 2008.

Vörös, Z., Baumjohann, W., Nakamura, R., Volwerk, M., Runov, A., Zhang, T. L., Eichelberger, H. U., Treumann, R., Georgescu, E., Balogh, A., Klecker, B., and Rème, H.: Magnetic turbulence in the plasma sheet, J. Geophys. Res., 109, A11215, doi:10.1029/2004JA010404, 2004.

Vörös, Z., Baumjohann, W., Nakamura, R., Runov, A., Volwerk, M., Takada, T., Lucek, E. A., and Rème, H.: Spatial structure of plasma flow associated turbulence in the Earth's plasma sheet, Ann. Geophys., 25, 13-17, 2007, http://www.ann-geophys.net/25/13/2007/. 